\title{
Análise de conceitos de química quântica nos livros didáticos de química do PNLD 2018
}

\author{
Mariana R. dos Santos ${ }^{1}(I C)^{*}$, Henrique do N. Coutinho ${ }^{1}(I C)$, Evelyn J. de L. Toledo $(P Q)$ \\ *marizz00108@gmail.com
}

${ }^{1}$ Laboratório de Pesquisas em Ensino de Química (LPEQ), Instituto de Química, Universidade de Brasília (UnB), Brasília - DF

Palavras-Chave: Análise de Livros, PNLD, Quântica

\begin{abstract}
RESUMO: As aplicações do termo Quântica tem se tornado cada vez mais comuns no dia-a-dia das pessoas, seja por meio de inovações tecnológicas diversas, seja por meio da incorporação de certos conceitos, muitas vezes de forma distorcida, ao imaginário popular (a chamada "Moda Quântica"). O estudo desse assunto na escola, portanto, é uma necessidade cada vez maior. Diante dessa realidade, este trabalho tem como objetivo analisar a abordagem de alguns conceitos-chave da Química Quântica nos livros de Química do Ensino Médio aprovados no PNLD 2018. Chegou-se à conclusão que, de forma geral, os conceitos apresentados são corretos, mas carecem de profundidade. Além do mais, algumas obras abordaram apenas um ou mesmo nenhum dos conceitos elencados. Afirmamos, assim, a necessidade de se dar uma maior atenção aos conteúdos de Química Quântica nos livros didáticos de química do PNLD.
\end{abstract}

\section{INTRODUÇÃO}

A Mecânica Quântica e, mais especificamente, a sua aplicação a problemas da Química, denominada Química Quântica, tem um papel de destaque no mundo moderno. Está presente, por exemplo, em lasers e LEDs, componentes de quase todos os equipamentos eletrônicos à nossa volta, além de seu papel essencial no desenvolvimento de aparelhos de ressonância magnética e outras tecnologias que ajudam a melhorar a qualidade de vida das pessoas. Nas palavras do grande filósofo da ciência Thomas Kuhn (KUHN, 2018, p. 122): "A transição da mecânica newtoniana para a quântica evocou muitos debates a respeito da natureza e dos padrões da física, alguns dos quais continuam até hoje.".

O estudo dos princípios da Química Quântica no Ensino Médio, portanto, é essencial para desenvolver a competência 1 da Base Nacional Comum Curricular (BNCC) para as Ciências da Natureza e suas Tecnologias, a saber:

Analisar fenômenos naturais e processos tecnológicos, com base nas interações e relações entre matéria e energia, para propor ações individuais e coletivas que aperfeiçoem processos produtivos, minimizem impactos socioambientais e melhorem as condições de vida em âmbito local, regional e global. (BRASIL, 2018, p. 553).

Além disso, há um crescente uso do termo "quântica" e seus derivados na cultura popular (sal quântico, coach quântico, medicina quântica, etc.) como forma de promover certos produtos e serviços que geralmente não tem nenhuma relação direta com a Quântica propriamente dita. Há, inclusive, proposta de uma ideia legislativa que proíbe 
o uso do termo "quântico (a)" em serviços não relacionados à Física e suas áreas (Portal e - Cidadania, 2019). Essa proposta foi encerrada sem receber o mínimo de 20 mil apoios para ser discutida no Senado e talvez até se tornar um projeto de lei (SOUZA, 2013), mas é um sinal da preocupação da comunidade científica brasileira com esse fenômeno.

Assim, um bom entendimento do que é a Quântica, suas potencialidades e limitações é essencial para que o aluno possa exercer plenamente a sua cidadania, conforme preza a Lei de Diretrizes e Bases da Educação Nacional (BRASIL, 1996): "Art. 22. A educação básica tem por finalidades desenvolver o educando, assegurar-lhe a formação comum indispensável para o exercício da cidadania e fornecer-lhe meios para progredir no trabalho e em estudos posteriores.".

O local mais propício para se obter esses conhecimentos é a escola, na qual, segundo Warthae Faljoni-Alário (2005), o livro didático é o principal norteador das práticas de muitos docentes. Assim, o objetivo desse trabalho é realizar uma análise dos conceitos de Química Quântica nos livros didáticos, mais especificamente nos livros do PNLD 2018 para o Ensino Médio, haja vista que, conforme apontado por Homrich et al. (2019), a análise desses livros pode dar acesso a um panorama geral de situações de ensino vivenciadas por docentes de todo o país.

Cabe aqui fazer a distinção entre conceito e conteúdo. Para da Silva e Silvestre (2008) conteúdo é "o conjunto de valores, conhecimentos, habilidades e atitudes que o professor deve ensinar para garantir o desenvolvimento e a socialização do estudante". Conceito, por sua vez, é "a definição de um determinado termo". Neste trabalho, o foco serão os conceitos relacionados à quântica e como eles são tratados nos livros de Química do PNLD 2018.

\section{MetodologiA}

Em um primeiro momento, foi feita uma leitura diagonal dos três volumes de cada uma das seis coleções de Química aprovados no PNLD 2018 a fim de localizar os conteúdos de Química Quântica. Seguiu-se, então, à análise da correção e forma de apresentação de conceitos essenciais dessa área, a saber: "quantização da energia", "orbital", "transição eletrônica", "números quânticos" e a própria palavra "quântica".

A fim de facilitar a referência às coleções em questão ao longo do texto elas foram numeradas da seguinte forma: L1 (REIS, 2016), L2 (MACHADO; MORTIMER, 2016), L3 (BRUNI et al. 2016), L4 (NOVAIS; TISSONI, 2016), L5 (CISCATO et al. 2016) e L6 (SANTOS; MÓL, 2016) conforme pode ser visualizada no quadro 01.

QUADRO 01: INFORMAÇÕES GERAIS ACERCA DOS LIVROS DE QUÍMICA DO PNLD 2018.

\begin{tabular}{|l|l|l|l|l|}
\hline LIVRO & TíTULO & \multicolumn{1}{|c|}{ AUTOR } & EDITORA & ANO \\
\hline L1 & Química & Martha Reis & Ática & 2016 \\
\hline L2 & Química & $\begin{array}{l}\text { Eduardo Fleury Mortimer e Andréa } \\
\text { Horta Machado. }\end{array}$ & Scipione & 2016 \\
\hline L3 & $\begin{array}{l}\text { Ser } \\
\text { Protagonista }\end{array}$ & $\begin{array}{l}\text { Julio Cezar Foschini Lisboa, Aline } \\
\text { Thaís Bruni, Ana Luiza Petillo Nery, } \\
\text { Rodrigo Marchiori Liegel e Vera } \\
\text { Lúcia Mitiko Aoki }\end{array}$ & SM & 2016 \\
\hline L4 & Vivá & $\begin{array}{l}\text { Vera Lúcia Duarte de Novais e } \\
\text { Murilo Tissoni Antunes }\end{array}$ & Positivo & 2016 \\
\hline
\end{tabular}




\begin{tabular}{|l|l|l|l|l|} 
L5 & Química & $\begin{array}{l}\text { Carlos Alberto Ciscato, Luis } \\
\text { Fernando Pereira, Emiliano } \\
\text { Chemello e Patrícia Proti }\end{array}$ & Moderna & 2016 \\
\hline L6 & $\begin{array}{l}\text { Química } \\
\text { Cidadã }\end{array}$ & $\begin{array}{l}\text { Wildson Santos e Gerson Mól } \\
\text { (coordenadores) }\end{array}$ & AJS & 2016 \\
\hline
\end{tabular}

\section{Resultados e Discussão}

Após análise dos livros didáticos, os conteúdos de química quântica foram encontrados no volume 1 dos livros L1, L2, L3, L4 e L5. O L6 possui o conteúdo nos volumes 1 e 3 . O quadro 02 mostra a ocorrência dos conceitos nas respectivas coleções.

Quadro 02: Ocorrência dos quatro conceitos - chave escolhidos nos livros analisados.

\begin{tabular}{|c|c|c|c|c|c|c|}
\hline & L1 & L2 & L3 & L4 & L5 & L6 \\
\hline $\begin{array}{c}\text { Quantização } \\
\text { da Energia }\end{array}$ & $\mathrm{X}$ & $\mathrm{X}$ & $\mathrm{X}$ & - & $\mathrm{X}$ & $\mathrm{X}$ \\
\hline Orbital & - & $\mathrm{X}$ & - & - & - & $\mathrm{X}$ \\
\hline $\begin{array}{c}\text { Transição } \\
\text { Eletrônica }\end{array}$ & $\mathrm{X}$ & $\mathrm{X}$ & $\mathrm{X}$ & - & $\mathrm{X}$ & $\mathrm{X}$ \\
\hline $\begin{array}{c}\text { Números } \\
\text { Quânticos }\end{array}$ & - & $\mathrm{X}$ & - & - & - & $\mathrm{X}$ \\
\hline Quântica & - & - & - & - & - & - \\
\hline
\end{tabular}

Com base no conteúdo analisado a partir dos livros didáticos aprovados no PNLD de 2018 e com o objetivo de analisar os conceitos, o primeiro conceito a ser discutido é o de Quantização da energia.

O L1 começa abordando o tema no capítulo 6, onde a autora utiliza a teoria de Max Planck: "Os corpos aquecidos emitem radiação não sob a forma de ondas, mas sob a forma de pequenos 'pacotes' de energia denominados quantum, ou seja, a energia é descontínua." (REIS, 2016, p.155).

A explicação da autora perante a citação foi que a energia não é propagada de forma contínua e sim na forma de pacotes. Foi definido também que cada quantum equivalia a uma quantidade definida de energia que era proporcional à frequência da radiação. Há uma imagem da representação de um feixe de luz ampliado mostrando o que deveria ser os quanta (quanta é o plural de quantum). 


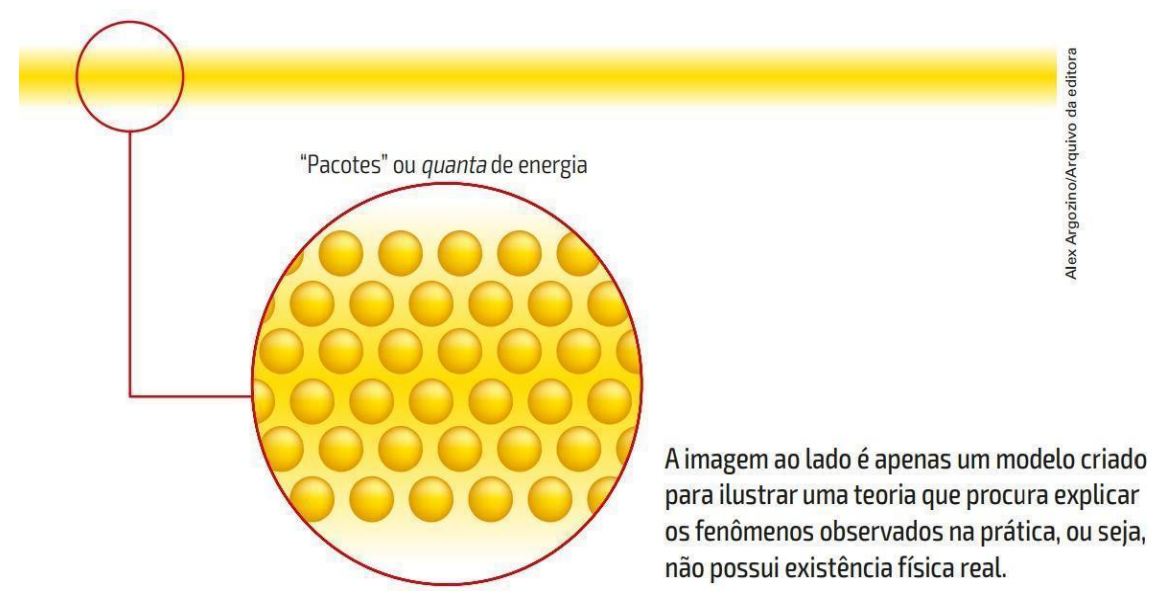

Figura 01: Feixe de luz ampliado, quanta de energia. Fonte: Reis (2016, p.155)

Esse conceito é retomado posteriormente no mesmo capítulo para exemplificar os postulados de Bohr: "Só é permitido ao elétron ocupar níveis energéticos nos quais ele se apresenta com valores de energia múltiplos inteiros de um fóton." (REIS, 2016 p.157).

No L2, o conceito também tem sua abordagem no capítulo 6 do livro e sua primeira definição vem acompanhada da hipótese quântica de Max Planck: "A hipótese quântica de Planck estabelece que a energia pode ser absorvida ou emitida apenas como a quantidade representada por hv (chamada de quantum) ou múltiplos inteiros desse valor." (MORTIMER; MACHADO, 2016, p.161).

O conceito volta a aparecer para resumir a apresentação dos postulados de Bohr, citando apenas que em seu modelo a energia é "quantizada".

$\mathrm{Na}$ análise do L3 foi possível observar que os autores utilizaram o conceito de energia quantizada durante a explicação do modelo de Bohr utilizando a também a teoria de Planck: "Segundo Planck, toda a energia do elétron é quantizada, ou seja, os elétrons absorvem ou emitem quantidades fixas de energia na forma de pequenos pacotes denominados quanta." (BRUNI et al. 2016, p.89).

Após análise do $L 4$, não foi observado nenhum conceito de quantização da energia.

No L5 o tema também é abordado durante a explicação do modelo de Bohr utilizando o trabalho de Max Planck para exemplificar: "Em 1900, o físico alemão Max Planck (1858-1974) sugeriu a hipótese da quantização da energia para exemplificar os resultados da radiação emitidas por sólidos aquecidos a várias temperaturas." (CISCATO et al.,2016, p. 94).

O L6 foi único a abordar o tema em dois volumes, sendo eles o 1 e 3 . O primeiro aborda a quantização no modelo atômico de Bohr de forma sucinta, já o terceiro possui um capítulo exclusivo para a abordagem da Química Quântica e começa a retratar o conteúdo no primeiro tópico do capítulo chamado "A Visão Clássica do Mundo Físico" após comentar sobre a Física Clássica e introduzir a Física Moderna, mencionando a teoria de Max Planck e o fato da radiação ser emitida e absorvida através de "pacotes" de energia. O assunto é retomado no tópico denominado "Modelo Quântico para o Átomo" onde os autores explicam a origem da Teoria dos Quanta passando pela propagação em forma de "pacotes de energia" e chegando na Mecânica Quântica.

Sobre o conceito analisado verificou-se que os livros L1, L2, L3 e L5 abordam o tema em sua literatura e também de maneira semelhante. O L4 por sua vez não aborda 
o tema em nenhum de seus volumes. O L6 foi o que mais abordou o assunto, incluindoo em 2 volumes e utilizando um pouco do contexto histórico.

O termo utilizado para explicar a quantização foi pacotes de energia, muitas vezes escrito entre aspas o que denota que os autores entendem que a expressão não é uma explicação literal. Embora essa compreensão possa ser clara para os autores nem sempre o será para os professores e seus alunos podendo ocasionar um obstáculo epistemológico (BACHELARD, 1996). Quantum significa quantidade em latim. Dizemos que uma grandeza é quantizada quando ela só pode assumir determinados valores múltiplos de um valor mínimo (um quantum).

Uma outra questão que devemos chamar atenção foi a representação imagética utilizada por Reis (2016, p.155). A autora, a fim de exibir o que seria os quanta de energia, optou por uma coloração amarela, apesar dos quanta de energia não terem o comprimento de onda da luz amarela. Essa escolha pode fazer com que o estudante associe a cor da luz com a cor dos quanta sendo um obstáculo epistemológico. Assim, ele poderá imaginar, por exemplo, que a luz branca tem quanta branco.

\section{O segundo conceito analisado foi o de Orbital.}

No L1 o conceito de orbital não é tratado. O L2 possui um tópico no capítulo 6 exclusivo para a abordagem do modelo atual contemplando o comportamento dual do elétron, incerteza e o orbital que é conceituado como: "A região mais provável de encontrar um elétron a certa distância do núcleo." (MORTIMER; MACHADO, 2016, p. 176).

Nos L3, L4 e L5 não foi encontrada definição para orbital. O L6 volume 3 assim como o L1 possui um tópico no capítulo 6 que se refere a função de onda e os orbitais atômicos. O mesmo é assim definido: "Orbital é a região no espaço em que há alta probabilidade de encontrarmos o elétron." (SANTOS; MÓL, 2016, p.252).

A expressão Orbital Atômico foi estabelecida pela primeira vez por Robert S. Mulliken (1932, p. 50) de maneira a abreviar "função de onda orbital para um elétron". Em sua conferência para o Nobel em 1966, ele conceitua o orbital como:

[...] o termo "orbital" é simplesmente uma abreviatura para a função de onda orbital para um elétron ou, de preferência, para autofunção orbital de um elétron. Esta última expressão refere-se a qualquer uma das chamadas soluções características ou autofunções de equação de onda da mecânica quântica de Schrödinger para um único elétron em um átomo ou molécula (MULLIKEN,1966, p.132).

Com o objetivo de interpretar o conceito de função de onda, Max Born em 1927 formulou a interpretação estatística da função de onda, que pode ser sintetizada em uma frase de Born "Resumidamente, é isto: as ondas são ondas de probabilidade" (BORN, 1935 p.157, tradução nossa) Essa ideia tem como base uma analogia com a óptica, sendo essa analogia afirmada por Max "[...] a analogia com a óptica sugere que uma certa expressão quadrática formada a partir da amplitude da onda deve ser interpretada como a força da corrente ou como o número de elétrons dispersos." (BORN, 1989, p. 95, tradução nossa).

Born continua dizendo: "Estes são os motivos da convicção da exatidão do princípio de associar a amplitude das ondas ao número de partículas (ou probabilidade)". (BORN, 1989, p. 95, tradução nossa).

Portanto, o conceito de orbital foi interpretado como densidade de probabilidade levando o interpretador a definir o orbital nos livros didáticos como sendo uma região de 
probabilidade de se encontrar o elétron, ou seja, uma versão completamente realista para algo complexo e absolutamente matemático.

\section{O terceiro conceito analisado foi o de Transição Eletrônica.}

O L1 trata o assunto ao falar sobre o modelo de Bohr, de maneira sucinta, sem muitos detalhes, como pode-se observar no trecho: "Se um elétron no estado fundamental absorve um fóton (quantum de energia), ele "salta" para o nível de energia imediatamente superior e entra num estado ativado (logo, numa situação de instabilidade)." (REIS, 2016, p.157).

O L2 elucida mencionando o teste de chamas e depois, para um melhor entendimento do conceito de "energia quantizada" o termo volta a ser mencionado com os postulados de Bohr.

Quando fornecemos energia ao átomo de hidrogênio, os elétrons podem saltar para níveis de maior energia. Ao retornarem ao estado fundamental, eles emitirão essa energia. Segundo o modelo de Bohr, só são possíveis certos níveis, correspondentes aos estados estacionários; assim, a radiação emitida tem frequência bem característica, constituindo o espectro de linhas. (MORTIMER; MACHADO, 2016, p. 176). o aprofunda.

O L3 também faz menção ao tema ao abordar o modelo de Bohr, entretanto, não

O modelo de Bohr explicava os espectros descontínuos dos elementos. Para ele, os elétrons que recebiam energia (proveniente do aquecimento ou de descargas elétricas) passavam para níveis de maior energia. Quando eles se encontravam nesses níveis, dizia-se que o átomo estava eletronicamente excitado. Ao retornarem ao estado de menor energia, denominado estado fundamental, acontecia a emissão de energia que poderia ocorrer sob a forma de luz. (BRUNI et al. 2016 p.90, grifo do autor).

O L4 não explica o conceito, apenas menciona que, para Bohr elaborar uma explicação sobre os elétrons, o mesmo cita que quando uma amostra contendo um determinado elemento recebe energia ele emite uma radiação luminosa. O L5 e L6 volume 1 descrevem o assunto através dos estudos de Niels Bohr.

Quando um elétron passa de um estado de energia elevada para um estado de energia menor, ele emite certa quantidade de energia radiante, sob forma de um fóton de comprimento de onda específico, relacionado com uma das linhas do espectro desse elemento. (CISCATO, 2016, p.158).

De acordo com o trabalho de Bohr, ao receber energia, os elétrons "saltam" para os níveis mais energéticos mais afastados do núcleo. Posteriormente, eles podem perder a mesma quantidade de energia. Quando isso acontece, eles emitem energia, "saltando" de volta para os níveis mais baixos. (SANTOS; MÓL, 2016 p.174)

Após análise dos livros, o termo "saltar" ficou extremamente evidente no vocabulário dos autores, sendo repetido inúmeras vezes, inclusive entre aspas e sem explicar por que esse recurso foi usado, deixando a encargo do leitor interpretar a razão da não literalidade do termo. Essa palavra utilizada para descrever a transição eletrônica configura-se como animismo, ou seja, atribui características humanas para descrever o comportamento do elétron. Se imaginarmos o movimento de um salto do elétron em um 
lóbulo do orbital p para outro lóbulo do mesmo orbital para que de fato seja um salto, o elétron teria que percorrer todo o caminho estando em regiões de probabilidade nula, 0 que não é possível. Assim, o uso no animismo não apenas dificulta o entendimento da transição eletrônica como oculta a existência do tunelamento.

Dessa forma, pudemos observar que nenhum livro descreve o fenômeno como Transição Eletrônica, quando esse conceito aparece é através da expressão Salto quântico.

\section{O quarto conceito analisado foi o de Números Quânticos.}

O L1 não conceitua o tema, apenas coloca o número quântico principal e o secundário entre parênteses ao explicar o modelo atômico de Sommerfeld e volta a mostrá-los ao explicar a distribuição eletrônica. O L2 possui um tópico para Números quânticos no capítulo 6 onde os quatro números quânticos são mencionados e descritos.

É importante lembrar que, no modelo de Bohr, apenas a energia total do elétron estava quantizada (energia apenas com valores discretos), o que resultava na ideia de níveis de energia associados a um único número inteiro, chamado de número quântico. (MORTIMER; MACHADO, 2016, p. 176, grifo do autor).

Não existe menção ao tema no L3, L4 e L5. O L6 possui uma conceituação geral do assunto.

Foi o físico austríaco Erwin Schrodinger [1887-1961] quem conseguiu propor uma solução matemática, para calcular os níveis de energia do elétron. Em sua abordagem, ele se apoiou na natureza ondulatória do elétron e para cada valor de energia propôs uma função de onda, representada pela letra grega $\Psi$ (psi), que permite chegar a três variáveis denominadas números quânticos: principal, secundário e magnético (SANTOS; MÓL, 2016, p.251, grifo do autor).

Os números quânticos surgem da resolução da equação de Schrödinger, sendo então uma dedução matemática muita complexa de ser explicada e compreendida por alunos do ensino médio. Porém, exceto pelo spin, cada um dos números quânticos tem um paralelo clássico permitindo que seja explicado teoricamente ao invés da abordagem memorística encontrada nos textos.

O número quântico principal (n) por exemplo, está diretamente relacionado com o "tamanho" do orbital, no sentido de que quanto maior o $\mathbf{n}$, maior a distância do núcleo atômico na qual ainda há uma densidade eletrônica considerável. Tanto é que muitas vezes ele é associado à ideia de "camada", herança do modelo semi-clássico de Böhr.

Os números quânticos secundários (I) e magnético $\left(\mathbf{m}_{\mathbf{I}}\right)$, por sua vez, são relacionados à "forma" dos orbitais (orbital s esférico, orbital p duplo ovoide e assim por diante) e sua orientação no espaço (se $\mathbf{p}_{\mathbf{x}}, \mathbf{p}_{\mathbf{y}}$ ou $\mathbf{p}_{\mathbf{z}}$, por exemplo), respectivamente.

O número quântico magnético de spin $\left(\mathbf{m}_{\mathbf{s}}\right)$, por fim, é relacionado de forma errada no ensino médio como sendo associado à rotação dos elétrons no seu próprio eixo, sendo indicado com uma seta para cima ou para baixo, assumindo valores +1/2 e $1 / 2$. Essa abordagem, porém, não é correta, pois a própria proposição da Equação de Schrödinger já supõe um comportamento ondulatório para o elétron e, portanto, não podemos atribuir-Ihe rotação, que seria uma propriedade de partícula, sem gerar uma certa imprecisão. Mais benéfico para o entendimento dessa propriedade por parte dos alunos seria a discussão das suas consequências no dia-a-dia, como o funcionamento dos ímãs permanentes, por exemplo, hoje tão comuns em vários aparelhos eletrônicos. 


\section{O último conceito analisado foi a palavra Quântica.}

A própria palavra quântica não é definida em nenhum dos livros didáticos analisados, mais uma vez deixando o tema em aberto e favorecendo a memorização dos outros conceitos da química quântica presentes nos livros que seriam muito mais fáceis de serem interpretados caso houvesse a definição da palavra no primeiro momento de estudo do tema.

O conteúdo de Química Quântica não é muito explorado nos livros didáticos. Porém, é um conteúdo de extrema importância para o futuro da humanidade, uma vez que está presente todos os dias no cotidiano de todos. Além disso, a tecnologia quântica é um campo relativamente novo no campo da engenharia, física, química e computação.

Utilizando métodos mecânico-quânticos é possível, por exemplo, modelar moléculas candidatas a cristais líquidos (GARCIA et al., 1999), os quais possuem diversas aplicações no cotidiano, como nas telas de LCD.

Algo que pode ser o avanço mais importante da história da computação está nas mãos da sociedade para que seja introduzido nas camadas mais jovens da população a fim de que não caia na escuridão e que esses jovens se interessem e busquem ainda mais conhecimento sobre o assunto.

O termo quântico vem sendo apropriado por outras situações, como a "cura quântica" que nada tem de ciência, mas se apropria de um termo que soa bastante científico para aumentar suas vendas. Conforme afirma Polkinghorne (2016, p. 109) "É evidente que a teoria quântica é estranha e surpreendente, mas não é tão esquisita a ponto de permitir que "vale tudo"

Assim, a fim de evitar que as pessoas sejam enganadas pela chamada "Moda Quântica" acreditamos que é necessário que o conteúdo seja abordado de forma mais adequada nos livros do ensino médio, de maneira a formar cidadãos com conhecimento necessário para julgar o que é certo e o que está sendo criado com o objetivo apenas de ludibriar os indivíduos por interesses escusos.

\section{CONCLUSÃo}

Essa pesquisa teve como o objetivo analisar os conceitos de química quântica abordados nos livros de química aprovados pelo PNLD 2018. Após a pesquisa realizada foi possível perceber que a maioria aborda o conteúdo apenas no primeiro ano do ensino médio, sendo a única exceção o livro Química cidadã que abordou o conteúdo nos volumes 1 e 3 . Nenhum dos livros preenche completamente os requisitos analisados nesta pesquisa, porém, dois dos seis livros contemplaram quatro dos cinco conceitos avaliados, faltando apenas a definição da própria palavra quântica.

A pesquisa também mostrou a dificuldade dos autores em apresentar adequadamente a teoria quântica, promovendo então um estudo por meio da memorização de conceitos.

Desta forma, conclui-se que é necessário trabalhar melhor os conteúdos de química quântica nos livros didáticos, evitando deixar brechas para que os conceitos sejam mal interpretados pelos alunos, levando-os a acreditar em ideias absurdas e sem nexo fomentadas nos dias atuais sob o nome da quântica. 


\section{AgRAdeCIMENTOS}

Universidade de BrasíLIA. PET - QUI/UnB. PET/SeSU/MEC.

\section{REFERÊNCIAS}

BACHELARD, Gaston. A formação do espírito científico: contribuição para uma psicanálise do conhecimento. Rio de Janeiro: Contraponto, 1996. 316 p.

BRASIL. Base Nacional Comum Curricular: Ensino Médio. Brasília: MEC/Secretaria de Educação Básica, 2018.

BRASIL. Lei de Diretrizes e Bases da Educação Nacional, LDB. 9394/1996.

BORN, Max. The Restless Universe. Blackie \& Son limited, 1935.

BORN, Max. Atomic physics. New York: Dover Publications Inc., 1989.

BRAGA, João Pedro. Fundamentos de Química Quântica. 1 ed. Viçosa: UFV, 2007. 272p.

CASSINELLO, Andrés; GÓMEZ, José Luis Sánchez. O Mistério Quântico: Uma expedição às fronteiras da física. 1 ed. São Paulo: Planeta, 2017. 271p.

CISCATO, Carlos Alberto, et al. Química. 1 ed. São Paulo: Moderna, 2016.

da SILVA, Ivaneide; SILVESTRE, Magali. O que são objetivo, conteúdo e conceito? Nova Escola, 2008. Disponível em: https://novaescola.org.br/conteudo/2755/o-que-saoobjetivo-conteudo-e-conceito\# $=$. Acesso em: 22 de Julho de 2020.

GARCIA, Edgardo et al. HFF: a force field for liquid crystal molecules. Journal of Molecular Structure: THEOCHEM, v. 464, n. 1-3, p. 39-48, maio 1999.

HOBSBAWM, Eric. Era dos extremos. 2 ed. São Paulo: Companhia das Letras, 2008. $562 \mathrm{p}$. 
HOMRICH, Alana; RUPPENTHAL, Nicolle; MARQUES, Carlos. Alimentação e o Ensino de Química: Uma Análise de Livros Didáticos Aprovados pelo PNLD 2018. Química Nova na Escola, v. 41, n. 1, p. 108-116, 2019.

KUHN, Thomas. A estrutura das revoluções científicas. 13 ed. São Paulo: Perspectiva, 2018. $324 \mathrm{p}$.

LISBOA, Julio Cezar Foschini, et al. Ser protagonista: Química. 3. ed. São Paulo: Edições SM, 2016.

MORTIMER, Eduardo Fleury; MACHADO, Andréa Horta. Química. 3. ed. São Paulo: Scipione, 2016.

Modelo de Bohr do hidrogênio. Khan Academy. Disponível em: https://pt.khanacademy.org/science/physics/quantum-physics/atoms-andelectrons/a/bohrs-model-of-hydrogen. Acesso em: 22 jul. 2020

MULLIKEN, Robert Sanderson. Electronic structures of polyatomic molecules and valence. II. general considerations. Physical Review, v. 41, p. 49-71, 1932.

MULLIKEN, Robert Sanderson. Spectroscopy, molecular orbitals, and chemical bonding. Nobel Lecture, p. 131-160, 12 dez. 1966. Disponível em:

https://www.nobelprize.org/prizes/chemistry/1966/mulliken/lecture/. Acesso em: 22 jul. 2020.

NOVAIS, Vera Lúcia Duarte de; ANTUNES, Murilo. Tissoni. Vivá - Química. 1. ed. São Paulo: Editora Positivo, 2016.

POLKINGHORNE, John. Teoria Quântica Uma Breve Introdução. 1 ed. São Paulo: L\&PM POCKET, 2016. 135 p.

Portal e - Cidadania. Ideia Legislativa - Proibir uso do termo "quântico(a)" em serviços que não relacionados a física e suas áreas. Disponível em: https://www12.senado.leg.br/ecidadania/visualizacaoideia?id=123420 Acesso em: 17 de fevereiro de 2020.

REIS, Marta. Química. 2. ed. São Paulo: Ática, 2016.

SANTOS, Wildson; MÓL, Gérson. (coord.) Química Cidadã. 3. ed. São Paulo: AJS, 2016.

SOUZA, Isabela. A Ideia Legislativa como ferramenta de participação política. Disponível em: https://www.politize.com.br/ideia-legislativa/ Acesso em: 17 de fevereiro de 2020. 
WARTHA, Edson José; FALJONI-ALÁRIO, Adelaide. A contextualização do ensino de Química através do livro didático. Química Nova na Escola, n. 22, p. 42-47, 2005. 